\title{
NOTAS
}

\section{OBSERVACIONES SOBRE LAS CONSTRUCCIONES CONDICIONALES EN LA HISTORIA DE LA LENGUA ESPANTOLA}

Una oración condicional, como todos sabemos, está compuesta de dos proposiciones; convencionalmente diremos que la primera enuncia la condición y recibe el nombre de prótasis, y que la segunda enuncia lo condicionado y se llama apódosis, aunque este orden diste mucho de ser obligatorio. La gramática tradicional decía que la primera era la subordinada y la segunda la principal. Por diversas razones, que iremos viendo, prescindiremos de la consideración de la condicional como par \{subordinada, principal\}. En cuanto a los términos prótasis y apódosis, hay una evidente posibilidad de confusión, ya que, en principio, se limitan a designar lo que va delante y lo que va detrás, de modo que en si Sócrates es hombre, Sócrates es mortal, la prótasis, en sentido estricto etimológico, es si Sócrates es hombre; pero, en Sócrates es mortal, si Sócrates es hombre, la prótasis, en ese mismo sentido es en cambio, Sócrates es mortal, al ser ahora lo que va delante. No obstante, como advertimos, y veremos en los autores aducidos en el texto, se suele igualar prótasis a "condición" y apódosis a "condicionado".

José Polo ${ }^{1}$ ha señalado, dentro de una línea interpretativa semántica, la relación de las condicionales con las causales: causa lógica, pero hipotética; en ellas, la actuación real como causa depende de la realización de la condición. Lidia Contreras ${ }^{2}$ por su parte, diferencia esta posibilidad en dos tipos: a) condicional propiamente dicha: si A, entonces $\mathrm{B}$; b) causales hipotéticas introducidas por si: Te lo digo por si acaso no lo sabes $=$ te lo digo porque acaso no lo sepas.

Esta distinción, por otro lado, permite rechazar la necesidad del argumento de María Luisa Rivero (véase infra, nota 10), en su intento de tratamiento generativo de las condicionales españolas, según el cual: "cuando una estructura condicional aparece incrustada en una oración cuyo verbo requiere un complemento en subjuntivo, ... la apódosis sólo puede aparecer en subjuntivo" (p. 210; en la ed. de 1977, p. 105).

1 José PoLo, Las oraciones condicionales en español (ensayo de teoria gramatical), Granada, 1971.

2 "El perfodo causal hipotético con si", BdFS, 11 (1959), 355-359; "Las oraciones condicionales", BdFS, 15 (1963), 33-109. 
Su ejemplo, en primer lugar, no parece válido metodológicamente, puesto que esconde una petición de principio (único caso en el que su afirmación se confirma, por otra parte): dudo que los otros, si lo supieran, actuaran de manera tan honrada, muestra, efectivamente, que actuaran es subjuntivo, verbo de la completiva de dudo, que exige subjuntivo, y, al mismo tiempo, condicionado o apódosis (convencionalmente) de si lo supieran. La presencia o ausencia de la condición no tiene nada que ver con que actuaran sea subjuntivo, hecho que sólo está determinado por dudo que. Es decir, el verbo de la principal selecciona el modo de la condicional cuando es completiva, afirmación que se nos antoja banal. En segundo lugar, puede incluso discutirse el valor de la condicional-completiva en estos ejemplos, pues si lo supieran podría interpretarse de modo harto compiejo, como una semi-causal hipotéticasemicondicional, cuyo carácter causal aparece en su interpretación consecutiva: como consecuencia de / en el caso de que lo supieran, y su carácter hipotético en la posibilidad de equivalencia con si acaso lo supieran. Incluso así, el coloquio restituye el indicativo si la presuposición es real, con lo que se hace patente el confuso terreno en el que se mueve la clasificación semántica de las oraciones compuestas.

No se puede olvidar tampoco que, en la simetría de sus dos elementos, ias condicionales proporcionan otra información: la rítmica, por una parte (véase García de Diego, cit. infra, nota 19), y la significativa, por otra, puesto que puede prescindirse, a veces, de la partícula condicionante o de otra expresión formal de la condicionalidad, como ya fue señalado por Eduardo Benot (Arte de hablar. Gramática filosófica de la lengua castellana, 4a ed., Madrid, 1921, pp. 311-312).

Las condicionales plantean, inicialmente, una serie de interrogantes, de los que procuraremos recoger algunos: a) Carácter de la relación entre sus elementos: por el tipo de juicio y por el tipo de condición. b) Evolución histórica, limitada al tipo más extendido, con si introductorio. c) Algunos aspectos de su problemática sincrónica, con exclusión de lo explicado en $a$ o, eventualmente, en $b$.

\section{CARÁcter de LA RELACión ENTRE LOS ELEMENTOS DE LA CONDICIONAL}

La oración condicional nos ofrece una secuencia de dos elementos (llamémoslos oraciones, como en la gramática tradicional, proposiciones, como parece imponerse hoy, incluso en manuales escolares, o sintagmas como hace J. Polo, op. cit., $\S 0.1$ ) relacionados por subordinación, coordinación, semicoordinación o semisubordinación. Como secuencia de dos acciones están relacionadas con las consecutivas, temporales, concesivas, modales y causales. Con estas últimas están también unidas porque expresan una relación de causa, que, en el caso de las condicionales, es hipótesis causal (ibid., $\S 1.5$ ), aunque no creemos (frente a Polo) que se trate sólo de una hipótesis causal lógica, sino también real, como hemos de ver. 
Precisamente desde el punto de vista lógico se han buscado clasificaciones de las condicionales por el tipo de juicios que las forman; hay, en efecto, dos juicios, cuya relación es siempre necesaria. Su estudio y tipificación podría partir hoy de la concepción kantiana de concepto de sujeto y concepto de predicado, para, tras la negación de la tesis del empirismo lógico de Carnap, llegar a una formulación de las concepciones de Kant, tal como hace J. J. Katz ${ }^{3}$. Para ello, el concepto de sujeto y el concepto de predicado son sustituidos por las nociones formales de lectura del sujeto y lectura del predicado. Por su especial carácter, las condicionales se prestan al estudio de la vinculación, como vemos en las condiciones necesarias para el mantenimiento del carácter analitico, contradictorio o sintético de los juicios que las componen. El desarrollo de este punto queda limitado, en estas páginas, a una indicación sobre los juicios analiticos.

La verdad o falsedad de un juicio analítico (expresado en una proposición) está en sus propios constituyentes: el predicado no añade nada a la comprehensión del sujeto; en proposiciones (expresiones de juicios) como los solteros no se han casado o los profesores son docentes, las notas de soltero y profesor (- casado, + docente, respectivamente) se repiten en las expresiones de los predicados correspondientes: al decirnos de un soltero que no se ha casado o de un profesor que enseña no se añade nada a lo que ya sabíamos. Para que una oración condicional mantenga la condición de analiticidad en toda ella, no basta con que las dos proposiciones que la integran sean expresión de dos juicios analíticos (no nos sirven ejemplos como si los solteros no se han casado, los profesores son docentes, salvo en su interpretación metalingüística), es necesario que exista entre ellos una vinculación, que consiste en que el sujeto de la primera proposición y el de la segunda comparten varios rasgos semánticos, y lo mismo sucede entre los predicados. Así, por ejemplo, se mantiene la analiticidad en si los solteros son hombres, los solteros son seres animados, con lo que vemos que la exigencia de coincidencia semántica se eleva a que haya un marcador semántico complejo común a un constituyente de la lectura del antecedente y a otro de la del consecuente (solteros 1 y 2 en nuestro anterior ejemplo).

La clasificación de las oraciones condicionales ha sido, y es, caballo de batalla y fuente de una serie de imprecisiones de quienes se han ocupado del tema ${ }^{3 b}$. José Polo (op. cit., \$ 3.1) distingue, por el tipo de condición, divisiones ternarias y binarias; de las ternarias, la de Lenz ${ }^{4}$, real, potencial e irreal, aprovechada por Harris 5 , tiene el inconveniente de que no diferencia, dentro de las reales, las categóricas u obligatorias de las contingentes. Parece necesario considerar tipos de

3 Filosofia del lenguaje, Barcelona, 1971, pp. 170 ss.

$3 b$ Cf. Francisco Calero y Marfa Jost Echarte, "Estudio estructural del sintagma condicional latino con la prótasis en indicativo", Durius, 3/6 (1975), 385-404.

4 Rodolfo LeNz, La oración y sus partes, Madrid, 1935.

5 M. HARRIs, "The history of the conditional complex from Latin to Spanish: Some structural considerations", $A L, 2$ (1971), 25-33. 
juicios junto a tipos de condición: un juicio hipotético, como si el sol calienta el tubo del termómetro, el mercurio sube, según Zaragüeta ${ }^{6}$, es una versión del categórico: el calor hace subir la columna de mercurio. El ejemplo anterior es una condicional real, distinta de las que podemos llamar reales-contingentes, porque del cumplimiento de la condición no se sigue necesaria y obligatoriamente el de lo condicionado, aunque se afirme la relación necesaria ${ }^{7}$ : no son versiones de juicios categóricos. Así, si vienes te veré, enunciado porque pensamos en una relación necesaria, no obliga físicamente, como el calor la subida del mercurio en el termómetro, y no se reduce a un juicio categorial como tu venida hará que yo te vea (a menos que se trate de situaciones excepcionales, como una curación psicológica de la ceguera neurótica, por ejemplo). Por esa razón desarrollaremos un tipo de clasificación más amplio, que reconocemos gustosos como deuda a las enseñanzas de Rafael Lapesa, en la Universidad Complutense, en cuanto a transmisión y codificación; en nuestro caso:

Reales: para el hablante (a veces, para el interlocutor, en "condicionales retóricas"), el cumplimiento de la condición comporta el de lo condicionado; aunque, de acuerdo con lo anteriormente expuesto, nosotros diferenciaremos los dos tipos, dependientes de que ese cumplimiento sea inevitable (reales-categóricas) o no (reales contingentes). En la lengua actual la cuestión es semántica, puesto que, formalmente, las dos construcciones son iguales, con verbo en indicativo en la condición:

reales categóricas: si llueve, se mojan las calles.

reales contingentes: si hace buen tiempo, iremos al campo.

Potenciales o contingentes: "ni se afirma si se niega la conexión entre la condición y lo condicionado" (GRAE, 433e), las dividiremos también en dos, según el mayor o menor grado de conexión:

contingente probable: Pero si Filis por aqui tornare; / hará reverdecer cuanto mirare. (Garcilaso, Egl. III) .

contingente dudosa: si pudiese ir, te lo llevaria gustosamente.

Adelantamos que, aunque históricamente diferenciadas, la dudosa no se distingue formalmente de la irreal no-pasado, que ahora veremos, a partir de la época latina, razón que favorece la confusión, y que la gramática normativa señala sin entender ( $G R A E, 434 c$, nota). La distinción que apoyamos evita, por otra parte, confundir la real-contingente si viene lo veré y la contingente probable si viniere lo veré (cf. portugués fà-lo-ei se ele vier), confusión que no logra evitar, por ejemplo, Martin Harris (1971).

Irreales o contrarias a la realidad: al plantearse el cumplimiento

6 Juan Zaragüeta, Filosofia y vida, Madrid, 1950, p. 50, cit. por J. Polo, $\$ 1.33$.

7 Real Academia Española, Gramática de la lengua castellana, Madrid, 1920. $\$ 433$ c. En adelante, GRAE. 
de la condición como imposible, se desprende que la relación entre la realidad y lo enunciado no existe. La diferencia que la lengua registra, en las dos clases de irreales, es la temporal, es decir, si el tiempo es o no pasado. Si el tiempo es pasado, la imposibilidad es absoluta y demostrada, si el tiempo es no-pasado, la diferencia entre la irreal y la contingente dudosa se borra hasta desaparecer, diacrónicamente, en la evolución de las formas, según veremos:

irreal no-pasado: si quisieras, lo harias.

irreal pasado: si hubieras querido, lo habrias hecho.

Como se ve, la triple división, para ser completa y explicar los trasvases de una categoría a otra, y la reducción de diferencias desde el latín, debe incluir, en cada grupo, una bipartición. Por supuesto, una vez señaladas las diferencias y puntualizaciones anteriores, podemos aceptar como base de trabajo la clasificación expuesta antes, o buscar una nueva, en cuyo caso los datos que pasamos a exponer tendrían que ser organizados de otro modo.

\section{Evolución histórica de LAS CONDICIONALES INTROdUCidAs POR "SI"}

Desarrollaremos los principales esquemas teóricos en los que se pueden distribuir los ejemplos de condicionales, en el paso del latín al castellano, y limitándonos a la peculiaridad de las formas con si, como suelen hacer, o destacar, la mayor parte de los que tratan el tema ${ }^{8}$. En cuanto al enfoque metodológico, esperamos mostrar, con lo que diremos a continuación, que el problema de las condicionales introducidas por si ha de estudiarse ligado al problema general del

S Emilio Alarcos, reseña al libro de Mendeloff, cit. infra, $R P h, 14$ (1960-61), 349-350; ANtonio Badía Marcarit, "El subjuntivo de subordinación en las lenguas romances y especialmente en iberorrománico", $R F E, 37$ (1953), 95-129; "Sobre las interpretaciones del verso 20 del Cantar de Mio Cid", $A O, 4$ (1954), 149-165; Mariano Bassols DE Climent, Sintaxis histórica de la lengua latina, t. 2, Barcelona, 1948; reed. Madrid, 1956, esp. 259-284; Sintaxis latina, Madrid, 1971, 2 ts.; EdouArd Bourciez, Eléments de linguistique romane, $5^{*}$ ed. Paris, 1967; A. Ernout y F. Thomas, Syntaxe latine, Paris, 1951, reed. 1963; Gustav GröBER, Grundriss der romanischen Philologie, t. 1, 2* ed. aumentada, Estrasburgo, 1904; M. HARRIs, "Systems or rules: A false dichotomy?", $A L, 3$ (1972), 83-93; Josef Herman, La formation du système roman des conjonctions de subordination, Berlin, 1963; R. KüHNER, y C. STEGMANN, Ausführliche Grammatik der lateinischen Sprache, 2 ts., cd. refundida por C. Stegmann, Hannover (1912-1914); J. M. LOPE BLANCH, "La expresión condicional en Diego de Ordaz (sobre el español americano en el siglo xvi)", $H R L(l)$, 379-400; H. MENDELOFF, The evolution of the conditional sentence contrary to fact in Old Spanish, Washington, 1960; W. MEYER LüBKE, Grammaire des langues romanes, III, Sytaxe, Paris, 1900; Felice MerLo, "La congiunzione sE e il sistema semantico dei periodi avverbiali", $R F$, (1957), 273-304; EMILIo NÁÑEz, "Sobre oraciones condicionales", ACerv, 3 (1953), 353-360; H. C. Nutring, "The Latin conditional sentences", Classical Philology, 7 (1926), 1-185; BERNARD PotTIER, reseña a Mendeloff en $B H i, 63$ (1961), 127-129; M. Ruch, "Objetivité et subịetivité dans la période hypothétique latine", $R R L, 14$ (1969), 101-109. 
verbo español. Otra consideración metodológica (en la que hay finalmente acuerdo en la polémica entre $M$. Harris y J. Green ${ }^{9}$ ) es la de que, en el paso del latín al castellano, es necesario suponer una evolución de la estructura profunda, pues lo contrario (en un modelo dependiente de la teoria tipica, ampliada) nos llevarla a negar la evolución lingüística, que se limitaría a la mera superficie.

Empezaremos nuestro examen diacrónico por el esquema teórico de las condicionales en latín clásico; tiempo habrá de ver las numerosas excepciones:

I. El hablante da por supuesto, subjetivamente, que la condición es real; si + indicativo / modo libre

$$
\begin{aligned}
& \text { si possum, id faciam (futuro de indicativo) } \\
& \text { si innocens est, profecto absolvetur (subjuntivo) } \\
& \text { si innocens est, eum absolvite (imperativo) }
\end{aligned}
$$

II. La hipótesis se presenta como contingente: los tiempos del modus potentialis, presente y perfecto de subjuntivo, empleados aquí, originan el término potencial.

$$
\mathrm{Si}+\underset{\text { perfecto }}{\text { presente }} \text { de subjuntivo/subjuntivo } \text { perfecto }^{\text {presente }}
$$

si possis, id facias (si puedas, lo hagas)

si potueris, id feceris (si pudieres, lo hicieres)

si + pluscuamperfecto de subjuntivo / -urus -eram si potuissem, id facturus eram (si pudiese, lo había de hacer)

III. La hipótesis se presenta como contraria a la realidad: irreal.

a) en el presente: si + imperfecto de subjuntivo / imperfecto de subjuntivo

si haberem, darem (si tuviera, diera)

b) en el pasado: si + pluscuamperfecto de subjuntivo / pluscuamperfecto de subjuntivo

si habuissem, dedissem (si hubiera tenido, hubiera dado)

9 J. N. GreeN, "Spanish conditionals: Systems or rules?", $A L, 3$ (1972), 75-85.

$\theta b$ Las traducciones latinas que incluimos son meramente orientadoras, puesto que, entendemos, es imposible traducir los matices latinos, al haberse perdido en español, como se verá. Si puedo lo haré; si es inocente, sea absuelto de verdad; si es inocente, absolvedlo. En los ejemplos listados posteriormente traduzco literalmente el tiempo y persona latinos. 
c) pasado con coloración de presente: si + pluscuamperfecto de subjuntivo/pluscuamperfecto de indicativo

\author{
Praeclare viceramus ... nisi Lepidus recepisset Antonium \\ (Cic. Epist. 12, 10, 3)* \\ Et si fata deum, si mens non laeva fuisset \\ impulerat ferro argolicas foedare latebras \\ (Virg. En. II, 54-5) ** \\ Perierat alter filius, si carnifici conviva non placuisset \\ (Séneca, De Ira, 2.33.6)
}

Fijémonos en este tercer tipo -SE / -RA, puesto que en él encontramos el antecesor del empleo subjuntivo de -RA, problema crucial en el desarrollo del castellano (cf. nota 94 de R. J. Cuervo a la Gramática de A. Bello).

Este tercer tipo de irreales, que llamamos acon coloración de presente», no debe confundirse, como hacen Harris y Bourciez, con la forma que puede sustituir a las contingentes (incluso a las reales contingentes), la perífrasis de futuro: amaturus eram, fórmula que ya aparece en Plauto (Cist. 154): si tacuisset, tamen ego eram dicturus, por si taceat (tacuerit), tamen ego dicam (dixerim) (si callase, yo hablaria, sin embargo). Se encuentra también en Ovidio (Tr. I, 7, 40) : emendaturus, si licuisset, eram, por si liceat (licuerit), emendem (emendaverim) (lo habría arreglado [arreglaría], si hubiese sido [fuese] libre de hacerlo).

Podemos decir, por tanto, que el latín rompe el esquema teórico con la combinación -SE / -RA y con el tipo -URUs; pero no son esas las únicas modificaciones, si bien las dos que citamos a continuación afectan a la conjugación castellana:

1) Confluencia del imperfecto de subjuntivo, futuro perfecto de indicativo, perfecto de subjuntivo, y nacimiento del futuro hipotético de subjuntivo castellano. Es fenómeno que aparece ya en latín arcaico, 186 a. J. C., en el Senatus consultus de Bacchanalibus, lin. 3: neiqvis eorum bacanal habvise velet sei ques / esent quei sibei deicerent necesvs ese bacanal habere eeis vtei ad pr vrbanvm / Romam venirent ("ninguno de ellos quiera celebrar la bacanal. Si algunos hubiere que dijeren que les era necesario celebrar la bacanal para sí, que vengan a Roma ante el pretor urbano"). El mismo fenómeno se da también en el valor contingente de este ejemplo de Ovidio (Elegia VIII, 5-6) : donee eris felix, multos numerabis amicos / tempora si fuerint nubila, solus eris ("si los tiempos fueren malos ..."), o en el Fuero de Madrid, XCIV (Crestomatia del español medieval, ed. Menéndez Pidal,

* "Venciéramos muy claramente ... si Lépido no hubiese recibido a Antonio".

* "Y si los destinos de los dioses, si la mente no hubiera estado errada, im. pulsara a manchar con el hierro los escondrijos argólicos".

* * "Pereciera el otro hijo, si el parásito no hubiera agradado al verdugo". 
I, 70): Et si alguno homine de conzeio dixerit: "mais le demos", pectet II morabetinos.

2) Completa evolución del sistema temporal clásico, con la formación de tiempos compuestos, primero del indicativo:

a) ser + participio de intransitivos o reflexivos La tienda es cogida; son idos.

b) haber + cualquier participio, que se complica con el valor transitivo de aver, en oposición con tener, para la posesión, con distinción de matices semánticos. (Cf. E. SEIFERT, "Haber y tener como expresiones de posesión en español", RFE, 17, 1930, 233-276; 345-389.)

c) haber + infinitivo, origen de futuro y condicional.

Esta evolución de los tiempos y formas temporales tiene dos consecuencias muy importantes:

La primera es que a los cambios de los tipos $a$ y $b$ (tiempos compuestos que sustituyen al perfectum latino) se debe el paso de la forma -RA al subjuntivo, primero como pluscuamperfecto, luego como imperfecto ${ }^{10}$. A lo largo de la Edad Media subsiste el tiempo en -RA con valor de pluscuamperfecto de indicativo: Cid, 1573: poco auie quel ganara / d'aquel rey; Cuento del Emperador Otas, XVII (Crestomatia, II) : el (Garssir) queria saber del emperador Otas por que le

10 D. BECKER, Die Entwickelung des lateinischen Pluscuamperfect Indicativus in Spanischen, Leipzig, 1928; V. BEJARANo, "Sobre las dos formas del imperfecto de subjuntivo y el empleo de la forma en -se con valor de indicativo", Strenae (Hom. Ga. Blanco), Salamanca (1962), 77-86; D. L. Bolinger, "On the -ra form", $H, 31$ (1948), 341-342; "Subjunctive -ra and -se: Free variation?", $H, 39$ (1956) , 345349; "One subjunctive or two?"; $H, 57$ (1974), 462-471; R. Davis, "A note on the - $r a$ indicative in fifteenth century Spanish", PhQ, 13 (1934), 218-220; G. T. Fisf, "The neglected tenses: hube hecho, indicative -ra, re", $H, 46$ (1963), 138-142; HARRI MeIer, "Sintaxis española, peninsular e hispanoamericana", $C H(3)$, 601-611; EMILIo RIDRuejo, "Cantaria por cantara, en la Rioja", Ber, 89 (1975), 123-134; M. L. RIVERo, “On conditionals in Spanish", GSRL, 196-214 (véase también Estudios de gramática generativa del español, Madrid, 1977, pp. 87-110); VIDAL LAmíuiz, "Cantara y cantase", RFE, 54 (1971), 1-11; JUAN M. LOPE BLANCH, "Algunos usos de indicativo por subjuntivo en oraciones subordinadas", NRFH, 12 (1958), 383-385; J. Mallo," "El empleo de las formas del subjuntivo terminadas en -ra con significación de tiempos de indicativo", $H, 30$ (1947), 484-487; "La discusión sobre el empleo de las formas verbales en -ra con función de tiempos pasados de indicativo", $H, 33$ (1950), 126-139; Robert F. SPAulding, "An inexact analogy. The -RA form as a substitute for the -RíA", $H, 12$ (1929), 371-376; C. N. Staubach, "Current variations in the past indicative uses of the ra form", $H, 29$ (1946), 355-362; LEAvITT $O$. WRIGHT, "The subjunctive forms in -ra and se in Spanish American speech", H, 9 (1926), 170-173; "Further remarks on -ra and -se", ibid., p. 201; "The indicative forms in $r a$ in Spanish America", ibid., pp. 288-293; "The indicative function of the -ra verb form", $H, 12$ (1929), 259-278; "The disappearing Spanish verb form in -re", $H, 14$ (1931), 107.114; The -ra verb form in Spanish, Univ. California, $M P h, 15,1,1932$; "The earliest shift of the Spanish -ra verb form from the indicative function to the snbjunctive: 1000-1300 A. D.", Lan, 9 (1933) , 265-268; "The -se verb form in the anodosis", $H R, 1$ (1933), 335-336; "Grammar slips in the new Spanisl constitution", MLF, 18 (1933), 96-97; "The Spanish verb form with the greatest variety of functions", $H, 30$ (1947), 488.495. 
non quesiera dar su fija, \& por que despreçiara su mandado; (ibid.): $e$ aprendiera bien el griego. Este uso llega a Mariana ${ }^{11}$, pero ya Juan de Valdés dice, criticando algunos aspectos del estilo del Amadis: no me suena bien viniera por avia venido, ni passara por avia passado ${ }^{12}$. Como pluscuamperfecto de subjuntivo, en correlación con el pluscuamperfecto perifrástico, y dentro del tipo -SE / -RA del que hablábamos arriba, tenemos en Berceo, Sta. Oria 152a,b: Si solo un poquiello me oviesen dexada, / grant amor me ficieran; y en Cervantes (Quijote, I, 51): Ni de su fin y acabamiento pudo alcanzar cosa alguna, ni la alcanzara ni supiera si la buena suerte no le deparara un antiguo médico, que tenia en su poder una caja de plomo.

La segunda consecuencia de la evolución de las formas temporales se debe al cambio del tipo $c$, es decir, haber + infinitivo, que origina, además del futuro, la fórmula cantare habebam, condicional, potencial o pospretérito, que viene a sustituir al tipo amaturus eram que veíamos arriba (Plauto, Ovidio), y al modus potentialis, primero en la consecuencia, de donde se extiende dialectalmente, $o$ en registros bajos (cf. Ridruejo; art. cit.) a la condición. En su origen se trata de una fórmula vulgar, tal como aparece en el Pseudo Agustinus, Sermones 253, 4; Sanare te habebat Deus per indulgentiam, si fatereris, donde ya estamos en el si confesares, te salvaria Dios...

Al establecer de nuevo el cuadro de las condicionales, observamos hasta qué punto cambia el esquema, salvo en el primer tipo, el de las reales, que permanece como en latín:

I. Hipótesis real: si + indicativo / modos y tiempos libres

(s)i quereedes oyr lo que uos quiero dezir, dizre uos lo que ui. (Disp. alma y el cuerpo, 1-2).

II. Hipótesis contingente. Hay una serie de restos del modus potentialis $^{13}$, desvirtuado por la combinación subjuntivo-indicativo:

$$
\begin{aligned}
& \text { Tanto fase un escudo } \\
& \text { entre él e la saeta, } \\
& \text { quanto el mundo pudo } \\
& \text { sy entre amos se meta }
\end{aligned}
$$

(Sem Tob, $104 a$ )

Es un ejemplo dudoso, por la rima (puede ser un ripio que obligue a elegir el subjuntivo). Al mismo tiempo es seguro, por la misma rima. No faltan ejemplos: $E$ pénalo gravemente si non se arrepienta

11 Como dice G. CIrot, Ẽtudes sur l'historiographie espagnole. Mariana historien, Burdeos, 1905.

12 Diálogo de ta lengua, ed. J. Montesinos, p. 175.

13 Véase Frede Jensen, y Thomas A. Lathrop, The syntaxi of the old Spanish subjunctive, La Haya, 1973, p. 179. 
(López de Ayaia, Rimado, 87d), en rima con parienta, sienta, afrenta, ofrece las mismas ventajas e inconvenientes que el anterior. En prosa, pero introducido por el sintagma por aventura, que codiciona el modo, tenemos este ejemplo del Fuero de Teruel $(403,4):$ Mas si por auen. tura el pendrador aquel ganado como es dicho non faga pregonar $e$ con él trasnochará et prouadol será, duplado como es fuero lo rienda.

Dejando a un lado estos restos con presente de subjuntivo (el perfecto de subjuntivo es tiempo de escasísimo empleo, según recuentos realizados para un estudio que tenemos en preparación), la hipótesis concebida como contingente se presenta en castellano medieval de estos modos:

a) Contingente probable:

$$
\text { si + tiempo en -RE } \begin{aligned}
& \text { / tiempo en -RiA } \\
& \text { / futuro o presente de indicativo } \\
& \text { / (que) + hortativa (presente del subjuntivo) }
\end{aligned}
$$

Si per mais apretaren los fiadores, cadat illis in periurio ( $F$. Madrid, XCIV).

Si vençieremos la batalla, creçeremos en rictad (Cid, 688).

Pero non vos enojedes si el pleyto se alongare (Rimado, 319a; con rima en -AR).

Jensen y Lathrop consideran excepcional la presencia del tiempo en -SE en estas construcciones, que podrían quedar así a caballo entre este tipo y el siguiente: $E$ si en alguna d'estas nouenas non pagasse segunt fuero, luego en aquella nouena el iudez lo prenga (F. Teruel, $20,4)$.

b.) Contingente dudosa:

$$
\begin{aligned}
& \text { / tiempo en -Ría } \\
& \text { si + tiempo en -SE / tiempo en -SE (si es subordinada) }
\end{aligned}
$$

En otros casos hay una tercera solución, unida a la pervivencia del futuro: si + futuro / exhortación. Ejemplos de este tipo aparecen en oración simple y compuesta, del primer tipo: Byue leda sy podrás (Juan Rodríguez del Padrón, Cancionero de Baena, $150 \mathrm{r}^{\circ}$, núm. 470) o precedido de por aventura: Si por auentura él uençra peche el homiżilio, con subjuntivo hortativo en la apódosis (Fuero de Teruel, 48, 5). Del segundo tipo, en período compuesto, y complejo, tenemos varios ejemplos: $E$ si el merino de Teruel con otro uezino baraiará por si mismo et el merino al uezino matare, peche el merino CCCC moravedis alfonsis (ibid., 26, 4). Precedidos de por aventura, con ese matiz hipotético que favorece el subjuntivo, como veíamos al principio de este 
estudio, tenemos varios ejemplos: Si por auentura la nodriça a su criado lech enferma dará e por aquella occasión aquel ynfant morrá, pagadas primera mientre las calonias, ixca por enemiga (ibid., 40, 2). $E$ si por auentura la muerte e la fidança de saluo ensenble negará $e$ ... non podrá seer uençido con testigos, salue se con XII uezinos (ibid., 48,6 ). (Véanse más ejemplos en Cantar de mio Cid, ed. Menéndez Pidal, Madrid, 1964, \& 155).

Creemos que esta solución pudo afianzarse en ciertos casos (como el texto legal del Fuero de Teruel, que tantos ejemplos ofrece) para evitar la confusión de la contingente dudosa (que conviene a un aséptico planteamiento legal) con la irreal-no-pasado, que, como veremos inmediatamente, coinciden formalmente. La abundancia de ejem. plos del Fuero atestigua la antigüedad de la construcción. Rafael Lapesa, en su edición parcial del Diálogo de la lengua (p. 45, nota 70), a propósito del vive leda si podrás que Valdés recoge (ed. Montesinos, p. 116; cf. también ibid., cap. 55, núm. 2), habla de influjo italiano. El italiano, en efecto ${ }^{14}$, desarrolla esta construcción, que aunque claramente atestiguada, en la lengua medieval, no impide una infuencia concreta en algún caso.

III. Hipótesis irreal

a) No-pasado (nótese que es formalmente idéntica a la contingente dudosa):

$$
\begin{aligned}
& \text { / tiempo en -Ría } \\
& \text { si }+ \text { tiempo en -SE / tiempo en -sE (si es subordinada) }
\end{aligned}
$$

Sy (este donzel) conmigo casase, él libraria esta tierra ante de un año (C. Emperador Otas, h. 1360-70, XVII).

b) Pasado:

$$
\text { si }+ \text { tiempo en -SE } \begin{aligned}
& / \text { tiempo en }-\mathrm{SE} \\
& \text { / tiempo en -RA }
\end{aligned}
$$

Sabet, si ellos le vidiessen, non escapara de mort (Cid, 2774).

Si a Millan croviessen, ficieran muy meior (Berceo, 288c).

(La forma simple, en ambos casos, tiene valor pasado: hubiesen visto, hubiesen creido; -RA es pluscuamperfecto de subjuntivo aún).

El esquema de las irreales se rompe ocasionalmente. Aparece el indicativo en la apódosis en ejemplos como: Et su hermano Miles mucho fue buen cauallero, si en si ouiese fe \& lealtad; mas mucho fue

14 Pavao Tekavcic, Grammatica storica dellitaliano, t. 2: Morfosintassi, Bolonia, 1972, $\$ 1306$. 
falso (Emp. Otas, XVII), donde aparece fue por fuera, y con valor de pasado (pluscuamperfecto), si hemos de hacer caso del segundo fue. Jensen (op. cit., $\S 181$ ) presenta varios ejemplos, que podríamos reorganizar haciéndolos arrancar de otros donde si no introduce propiamente una condicional, sino que se limita a la presentación de situaciones de hecho: mas si él fue brauo, no falló flaco al otro (Amadis, $200,85)$, o de empleos contingentes con verbos en indicativo, como el que recogen Jensen y Lathrop (op. cit., p. 81), sin precisar su valor y, en consecuencia, diferenciarlo: si no lo hazian, descaueçabalas ( $\mathrm{Ama}$ dis, 56, 510). De ahí podemos pasar, en teoria, a construcciones mixtas, con prótasis en subjuntivo y apódosis en indicativo (como el del Cuento del Emperador Otas que acabamos de citar): si tu quissiesses $e$ fuesse tu plaçer. En mi esti iudiçio non debie pereçer (Berceo, Milagros, 786c), o este del Poema de Alfonso XI (263): si más durara el mal perdida avia ssu tierra aquel rey, con durara como pluscuamperfecto de subjuntivo.

Harris (art. cit. de $A L$ ) insiste en la defensa de Mendeloff frente a la Historia de la lengua de Lapesa, de la posibilidad de -ría para la apódosis. Esto sólo es cierto en el no-pasado; para el pasado no parece adecuado el ejemplo que propone:

"Qual ventura serie esta, si ploguiesse al Criador / que assomasse essora el Cid Campeadorl" (Gid, 2741-42), que no es pasado, y que podría estar en el límite entre condicionales y desiderativas (latín si y sic) del famoso verso 20 , aunque debe tratarse aquí de una fórmula, puesto que, más adelante (Cid, 2750) volvemos a encontrarnos con la construcción: ¿Qual ventura serie si assomas essora el Cid Roy Diaz!, y en el Auto de los Reyes Magos, $63{ }^{15}$ : Nos imos otrosi, sil podremos falar. Jensen y Lathrop, por su parte, no añaden ningún ejemplo que pueda interpretarse como pasado; antes bien, parece ser no-pasado en todos sus ejemplos: Si vos viesse el Gid sanas e sin mal, todo serié alegre (Cid, 1402); sy esto te negassemos fariamos muy grant mal (Berceo, Sto. Domingo, 137c); e incluso a fines de la época medieval, en la Celestina $(272,15)$ : Si mi espada dixesse lo que haze, tiempo le faltaria para hablar.

De los esquemas anteriormente expuestos, y sus modificaciones excepcionales, más o menos frecuentes, podemos extraer una serie de consecuencias:

1) Ha desaparecido la diferencia formal entre la contingente dudosa y la irreal no-pasado, como consecuencia de la pérdida del uso de presente $y$, sobre todo, perfecto de subjuntivo en la contingente. La extensión al subjuntivo de la contingente probable con el nuevo tiempo en $-\mathrm{RE}$ (heredero parcial del perfecto de subjuntivo latino amaverim), y la extensión de la forma - $\mathrm{SE}$, mucho más empleada, que sustituye a la forma $-\mathrm{RE}$ en casi todos los casos donde sería etimológica-

15 Cf. Ricardo Senabre, "Observaciones sobre el texto del Auto de los Reyes Magos", AO, 27 (1977), 417-432. 
mente esperable (-REM latino, imperfecto de subjuntivo), y se extiende a otros, como vimos en si non pagasse, ... el iudez lo prenga $(F$. Teruel, 20, 4), donde todavía domina -RE en la Edad Media, consecuen. cia de que la forma -SE ya no es pluscuamperfecto de subjuntivo, sino el nuevo imperfecto. Los tratadistas, como Mendeloff y Merlo, coinciden en la necesidad de considerar conjuntamente la contingente dudosa y la irreal no-pasado, por la dificultad de separar los ejemplos de ambas clases, formalmente idénticos, insistimos.

2) La forma -Ría no aparece en la consecuencia de la condición que expresa hipótesis irreal pasada.

3) La forma -RA se introduce en el sistema precisamente en la consecuencia de la irreal pasada.

4) La forma -SE tiene tres usos, para los cuales el latín empleaba tres tiempos distintos:

\begin{tabular}{lll} 
Latin & $\begin{array}{c}\text { Valor en } \\
\text { castellano }\end{array}$ & \multicolumn{1}{c}{$\begin{array}{c}\text { Ejemplos } \\
\text { cast. con -SE }\end{array}$} \\
\hline $\begin{array}{l}\text { presente, perfecto } \\
\text { o imperfecto de } \\
\text { subjuntivo }\end{array}$ & $\begin{array}{l}\text { contingencia } \\
\text { dudosa }\end{array}$ & $\begin{array}{l}\text { sis' podiessen ir, } \\
\text { ferio yen. }\end{array}$ \\
\hline $\begin{array}{l}\text { imperfecto de } \\
\text { subjuntivo }\end{array}$ & $\begin{array}{l}\text { irrealidad } \\
\text { no-pasado }\end{array}$ & $\begin{array}{l}\text { si conmigo casase, } \\
\text { libraría esta tierra. }\end{array}$ \\
\hline $\begin{array}{l}\text { pluscuamperfecto } \\
\text { de subjuntivo }\end{array}$ & irrealidad & pasado a Millán croviessen, \\
\hline
\end{tabular}

5) Este sistema medieval inicial presenta más analogías con el moderno que el sistema del Siglo de Oro, que se plantea así como una interrupción entre el medieval y el actual.

Para llegar al sistema del Siglo de Oro se han producido, especialmente a partir del siglo xiII y, sobre todo, en el xIv, tres procesos:

1) Crecimiento de los tiempos compuestos de subjuntivo, que no aparecen en el Cid, pero sí en Berceo, aunque escasos: Si muerta me oviessen, ovieranme guarida (Berceo, Duelo, 17d), con concordancia del participio y el objeto directo. Estos tiempos compuestos pueden alternar con los simples, en lugar de los tiempos del perfectum latino, como en el ejemplo ya citado: si solo un poquiello me oviesen dexada, / grant amor me fiçieran (Berceo, Sta. Oria, $152 a, b$ ).

2) Extensión del compuesto en -RíA, atraído por el desarrollo de los tiempos compuestos del subjuntivo, en relación con los cuales lo tenemos en este ejemplo: Si non fuesse Siagrio tan adelante ido, / si oviesse su lengua un poco retenido, / non serie enna ira del Criador caydo (Berceo, Mil. 70a,b,c), donde tenemos dos tiempos compuestos de verbos intransitivos, construidos con ser como auxiliar, según uno de los dos sistemas medievales. 
3) Extensión del empleo de -RA, sobre todo a costa de -SE, que llega a perderse en la consecuencia. Interviene aquí la tendencia del periodo hipotético a la uniformación rítmica, ya señalada varias veces: el tiempo o forma temporal empleada en uno de los miembros se contagia al otro. El empleo de -RA se extiende a partir de la única posibilidad que tiene en principio: la consecuencia de las irreales pasadas en alternancia con -sE.

El primer caso fechado de -RA en la condición está en el Alexandre: Bien andante fuera Poro sy todos fueran atales (P2061d) (pero Bien andante fura Poro se todos fussen tales, $O, 1919 d$ ), con tiempo simple, pero valor de pluscuamperfecto. También en el Alexandre: Tenie que so preyto ouiera bien complido, / Se a Poro ouiera consigo retenido $(O, 1933 c, d)$, con tiempo compuesto, y, con tiempo simple, en el mismo manuscrito $O, 124 d$ : Se podiera Nicholao repentirase de grado, como irreal pasada igualmente. También del siglo xin, E. Ridruejo da este ejemplo de paso a la contingente: Sy tan buenos non fueran oy serien oluidados ( $F$. González, 355c), algo más próximo a la irrealidad que este otro, en el cual incluso el empleo de los tiempos nos acerca a la real: "E diol otrossi estonces esta ley: ... de si que coniurasse a ella e dixiessen así: que si ella tuerto non fiziera a su marido nin auie part en aquello quel dizien, quel non nuzrrien aquellas aguas amargas" (Gen. Estoria, I, $616 b$ 2-4). Rafael Lapesa (información verbal) tiene ejemplos de contingente en el Fuero Juzgo; el uso crece a lo largo de la Edad Media: E. Náñez (art. cit.) cita un ejemplo del Arcipreste de Hita:

Mi señor el Amor, si él a mi criera, El conbid de las monjas, aqueste rresçibiera. Todo viçio del mundo, todo plazer oviera: Sy a dormitorio entrara, nunca s'arrepintiera.

Nosotros hemos recogido el siguiente en el Dezir a Pero López de Ayala, de Pero Ferruz (h. 1380) (Crestomatia, II, 463) :

1-5 (ca) don Carcos non la poblara,

1.6 si tan grant mjedo tomara

1.7 de yelo, quai vos tomades.

En el Siglo de Oro, en Cervantes -RA llega a igualar a -sE, en la condición; E. Náñez y Criado de Val han recogido buen número de ejemplos. En la generación posterior a Cervantes domina -RA, lo que supone la disminución de -sE hasta nuestros días, en los que sólo parecen conservar predominio de -SE sobre -RA los asturianos y gallegos (apoyados por su valor indicativo de -RA, que limita su uso en condicionales), o los escritores de gusto arcaizante, como Larra y Valera. El uso hispanoamericano, al parecer, casi reduce -SE a variante estilística. En cuanto a lo condicionado (apódosis), -SE ha desaparecido ya 
a fines del xvi, aunque hoy queden restos vulgares, y una nueva extensión de reajuste rítmico que veremos más adelante.

El aumento de -RA se produce también, en los Siglos de Oro, a costa de -Ría. La colisión está ya en la Vida de Santo Domingo de Berceo, en quien, según nos comunica amablemente E. Ridruejo, no parece haber más ejemplos que éste: si bien no lo conplieres, mucho más te valiera (contingente probable). La confusión se opera en las dos direcciones. Ante las formas correctas, -SE / -RíA para contingente dudosa e irreal no-pasado, y -SE / -RA para la irreal en el pasado, aparece -RA en la apódosis de la contingente dudosa y la irreal en el no-pasado, mientras que en la irrealidad pasada aparece el condicional compuesto o antepospretérito, habria -do. En los Siglos de Oro se impone, sin embargo, -RA sobre -RíA, en la apódosis, naturalmente, lo que origina el típico esquema si tuviera, diera, preferido por los clásicos, que llega hasta el xviII en poesía. Pasado el Romanticismo decimonónico, donde subsiste por arcaísmo, la decadencia es vertical, en función de la nueva fórmula: si tuviera, daría.

Estos procesos alteran el sistema que hemos llamado medieval, de modo que dan la razón a la crítica de Alarcos a la separación tajante de Mendeloff entre el siglo xin y el xiv, frente a Pottier, que estaba de acuerdo con ella. Hay un movimiento de formas que Harris representa con un esquema que corregimos, puesto que en él figura la forma -Ría en lo condicionado-pasado, lo cual es inexacto, bien error por -sE, bien creencia en la posibilidad de -Ría en la apódosis irreal en el pasado, que discutimos antes. El esquema, para las irreales, parece ser el siguiente:

\begin{tabular}{l|c|c}
\cline { 2 - 3 } & $\begin{array}{l}\text { PROTASIS } \\
\text { (condición) }\end{array}$ & $\begin{array}{l}\text { APODOSIS } \\
\text { (condicionado) }\end{array}$ \\
\hline No-pasado & -se & -ria (-se) \\
\hline Pasado & -se & ra \\
\hline $\begin{array}{l}\text { Nuevos } \\
\text { tiempos }\end{array}$ & $\begin{array}{l}\text { hubiera can } \\
\text { hubiese cantado }\end{array}$ \\
\hline
\end{tabular}

Lo arriba expuesto afecta a las irreales y a la contingente dudosa, que tiene la misma forma que la irreal del no-pasado. La contingente probable ofrece la pervivencia de la forma $-\mathrm{RE}$ en la prótasis, aunque reducida por el empuje de -RA, como hemos dicho al hablar de esta segunda forma, y con la posibilidad, recogida como excepcional, de sustitución por -sE. Así, Juan M. Lope Blanch documenta -re como muy empleado en Diego de Ordaz coetáneo de Juan de Valdés ${ }^{16}$.

16 Según Lope Blanch -RE se usa 33 veces: (25 simples y 8 compuestas), siempre en la prótasis, frente a 11 -RA (10 simples, 1 compuesta), 10 -sE ( 9 simples, 1 compuesta) y dos -Rís, simples ambos. (Para -SE véase V. Bejarano, art. cit., y

L. Wright, "The se verbe form ..."). 
Germán de Granda ("Formas en -RE en el español atlámico y problemas conexos", $B I C C, 23,1968$ ) ha delimitado el área de pervivencia de formas del futuro hipotético de subjuntivo ( $\mathrm{RE}$ ), aunque es justo reconocer que, en general, sus funciones han sido absorbidas por el presente de indicativo o por el imperfecto de subjuntivo. Cuando se usa el indicativo (si llega tarde, que no reclame) la hipótesis tiene mayor fuerza expresiva de realidad. En cambio, el subjuntivo expresa contingencia problemática: si llegara tarde, que no proteste ${ }^{17}$.

Para finalizar esta panorámica diacrónica, aunque nos hemos limitado inicialmente al si introductorio, queremos recoger unos datos de Jensen y Lathrop sobre otras partículas condicionales. Como advertencia general, observan los autores que, mientras si parece predominar como introductor del primer elemento de la condicional (prótasis en el pleno sentido etimológico), las condiciones introducidas por otras partículas - locuciones tienden a construirse después de la enunciación de lo condicionado, aunque no sea obligatorio tal orden. Sería interesante realizar recuentos al respecto. También afirman que la interdependencia entre condición y condicionado es mayor cuando la partícula es si, y que la norma general para los verbos, con estos otros introductores, es el subjuntivo. Los ejemplos que aportan confirman esa norma, muestran otros matices junto al condicional (causal, temporal, concesivo, modal, principalmente) y ofrecen estas conjunciones, adverbios - locuciones conjuntivas: tal que, por tal que, con tal que, con tanto que, con, a, so (tal) condición que, en dado que, solo que, menos que, puesto que, y puesto (caso) que. Las dos últimas admiten indicativo, para "acontecimientos objetivos en el pasado", con el valor causal que se aprecia en ejemplos como: Puesto que todo esto falló bien conçertado I Pensaba luego en ál (Rimado, 648a); y puesto caso que yo no auia menester muchas salsas para comer, todauia me holgaua con las cortezas del queso (Lazarillo, 136, 4). También cuando, por último, puede introducir una condición: Cuando fuese del Demonio, todo seria al contrario (Sta. Teresa, Las moradas, 187, 4).

\section{Algunos aspectos de su Problemática sincrónica}

En este apartado cabría considerar tres problemas: la descripción de los varios modos de introducir una condición, el esquema actual de las construcciones condicionales, de modo amplio, y un intento de explicación de las mismas.

El primer punto, al que hemos aludido inicialmente al hablar de Benot y señalar, de paso, la posibilidad de condicionales sin nexo, que es uno de los modos de presentarse, cuenta con el trabajo clásico de Kany, y con la aportación de José Mondéjar ${ }^{18}$, para quien "es la for-

17 Cf. FISH, art. cit., y L. WRIGHT, "The disappearing Spanish verb form in -re". 18 Charles E. Kany, "Condition expressed by Spanish de plus infinitive", $H$, 19 (1936), 211-216; José MoNDÉJAR, "La expresión de la condicionalidad en español (conjunciones y locuciones conjuntivas)", RFE, 49 (1966), 229-254. 
ma verbal la que condiciona el empleo de tal o cual elemento conjun. tivo". Los giros condicionales estudiados por Mondéjar se dividen en cinco grupos: infinitivo precedido de $a$, de, con, con sólo, por, por ṫal de (no), con tal de (no); Gerundio (+ que); participio en construcciones absolutas; fórmulas condicionales: en tal caso, sin + sustantivo o pronombre, de otra suerte, otramente, donde no, de lo contrario; $y$ ante la condicionada, condicionante sin partícula o fórmula introductoria.

En cuanto al esquema actual de las condicionales con si, podemos presentarlo de este modo:

REALES (categóricas y contingentes) :

Condición: Indicativo (menos el antepretérito), los futuros y los pospretéritos.

Condicionado: Imperativo, indicativo (menos el antepretérito), subjuntivo, menos los futuros.

conTINGENTES (tienden a perderse, con restos esporádicos, salvo zonas dialectales, analizadas por G. de Granda) :

Condición: futuro o antefuturo de subjuntivo (hipotético).

Condicionado: presente o copretérito de indicativo, pospretérito.

IRREALES (en el no-pasado pueden expresar la contingencia, a veces combinadas con indicativo en la apódosis) :

Condición: no-pasado: pretérito de subjuntivo / pasado: antepretérito de subjuntivo.

Condicionado: pospretérito (-RíA) pretérito de subjuntivo (-RA) / antepospretérito (HABRía -Do) antepretérito de subjuntivo (-RA).

La ruptura de estos esquemas, como podemos ver en este ejemplo cervantino: Bien habrias dicho, hija, si la malicia ordinaria no se opusiera a tu discreto discurso, es antigua. Algunos empleos constituyen sistemas paralelos con abundante ejemplificación; es lo que sucede, como advertíamos en el título de las irreales, con la presencia de indicativo en la apódosis. Este uso es documentado por Lenz, Gili Gaya, Pottier, los tratadistas que se han ocupado de la temporalidad verbal, como C. Hernández y G. Rojo, y la Academia (1973, § 3. 14. 3) : Se perdia bien poca cosa si se muriera (S. y J. Alvarez Quintero, Doña Clarines). La Academia llama "imperfecto desrealizador" a esta fórmula si -ra / -aba, si llegara tu padre, nos marchábamos, que se extiende también al antecopretérito (pluscuamperfecto): si hubiera marcado Amancio, habia ganado el Madrid. La construcción puede parangonarse con la latina que hemos llamado de pasado "con coloración de presente": viceramus, nisi recepisset, y se explica fácilmente en términos semánticos, por atracción a la realidad de la expresión.

También recoge la Academia ( $\$ \S 3.14 .10,3.15 .7$ ), el empleo usual hoy, en ciertas construcciones, de la forma hubiese en la apódosis, en vez de hubiera. Como veíamos en el cuadro evolutivo, las formas en -SE de la apódosis caen, en un proceso consumado a fines del xvr, 
y si vuelven a aparecer hoy es consecuencia del intercambio de -RA y -sE, que tiende a hacerse total. Así tenemos fórmulas normativamente incorrectas, e históricamente de evolución paralela reciente, como: si hubieses querido te hubiesen pagado en el acto, donde para muchos hablantes ya no hay conciencia de que lo correcto es sólo te hubieran pagado, o si hubieras querido, te hubiésemos invitado, en vez del normativo te hubiéramos, tal vez ultracorrección, motivada por el deseo de diferenciar -RA/-SE y no repetir -RA/-RA. Esto no puede suceder en las formas simples correspondientes, porque la apódosis con -RA es prácticamente desconocida. Como casi nunca se oye si quisieras te pagaran, no hay motivo para que se diga si quisieses, te pagasen.

García de Diego ${ }^{19}$ explica el contagio (como el que por analogía vemos en si queria, hacia, por si quisiera, haria, o si querria, haria, misma interpretación), por la tendencia a la unificación rítmica. Tam. bién abundan, por otra parte, los ejemplos del coloquio en los que se rompe el esquema. Quizá el más antiguo de este tipo se encuentre en la Celestina, acto IV: Si no voy, ¿qué dirá Sempronio? que en realidad expresa la contingencia: si no fuese, qué diría. Este empleo para la contingente no es raro, pero he aquí otra muestra, que aparece en Realidad de Galdós: Si me dice pitos le contesto flautas ("si me dijera, le contestaría"). La ruptura del esquema llega incluso a formas de reales para las irreales: si estoy alli no pasa eso, por el pasado: si hubiera estado ... no hubiera pasado.

Dejemos aquí la consideración meramente descriptiva de la oración condicional, en la que hemos de tener en cuenta su situación entre las llamadas subordinadas ${ }^{20}$, y una serie particular de limitaciones, derivadas de su relación con otros tipos (causales, por ejemplo), de los distintos valores de $s i$, o de las dificultades de mero análisis semántico de la oración compuesta, como señalamos inicialmente, puntos desarrollados por Lidia Contreras (1963), o por José Polo (1971). Tampoco podemos olvidar que, dentro de su peculiar concepción del tiempo verbal en relación (mejor: sin relación) con el tiempo real, Weinrich ${ }^{21}$ habla de la dificultad que puede entrañar su clasificación (en francés se distinguen a veces con dificultad de las temporales), señala el carácter lógico de la relación, que motiva el rigor (relativo, como vemos) en las leyes de concordancia. Puesto que Weinrich trabaja sobre el francés, su estudio nos interesa principalmente por lo que tiene de general, que, además de lo ya resumido, es la observación de que la concordancia temporal mantiene la coherencia de tiempos del mundo narrado y tiempos del mundo comentado, más fácil de probar en francés por el empleo del copretérito (imparfait) en la prótasis del llamado modus irrealis (contingente o irreal no-pasado, para nosotros,

19 Vicente Garcia de Diego, "La información rftmica en las oraciones condicionales", EMP, 3 (1952), 95-107.

20 Véase G. Carrillo Herrera, "Estudios de sintaxis: las oraciones subordinadas", BdFS, 15 (1963), 165-221.

21 Harald Weinrich, Estructura y función de los tiempos en el lenguaje, Madrid, 1968. 
de modo más limitado y preciso). Weinrich se ve obligado a negar el carácter de realidad, potencialidad o irrealidad en las condicionales; no hay modos de realidad -dice-, sino oraciones que tienen en común el sistema metafórico temporal: no importa cada uno de los tiempos, sino el grupo temporal. En francés resulta bastante claro, en español no tanto, y se ve obligado a admitir que "las formas del subjuntivo y las metáforas temporales se reparten ... la función de limitar la validez del discurso" (p. 186); el intercambio de formas con el que trata de salvar la situación, con una vaga referencia a Gili Gaya, es inexacto. El recurso final de Weinrich a la historia, interesante en el caso del francés, de admitir su explicación, no es válido en español, donde la lengua actual se limita a desarrollar una posibilidad medieval, interrumpida por el uso excesivo de -RA en los Siglos de Oro, y no supone un ciclo consumado, como podría ser en francés.

Desde otro punto de vista, M. L. Rivero (art. cit.) ha intentado explicar la estructura profunda o representación semántica de la oración condicional, a partir de la pregunta de qué es lo realmente aportado, que puede reducirse mucho, como veremos, según la propia autora. M. L. Rivero propone, en síntesis, que la forma si es un verbo profundo del tipo creador de universos, y trata de probarlo con una serie de ingeniosas conmutaciones (hendiduras, pronominalización de oración, verbos factivos, interpretaciones irreales, transporte de la negación, consideración de mundos, supresión, incrustación y sinonimia); en todos esos casos cree encontrar que la prótasis (condición) tiene un carácter nominal, dependiente de un nudo $\mathrm{sN}$, y la igualdad de si y verbos como imaginar o suponer. Parece innecesario entrar en la crítica de sus argumentos, que no tienen siempre la misma fuerza probatoria $y$, a veces, son difícilmente aceptables, porque la autora, al final de la primera parte de su trabajo, nos da la clave, al decirnos que, para ella, es lo mismo que si sea un verbo o que la condicional dependa de un verbo creador de universos de tipo abstracto; sólo es cuestión de nombres. Viene a ser, pues, un nuevo dar vuelta a cuestiones ya expuestas, con planteamientos atrevidos e, innegablemente, atractivos por su ingenio. Creo que esas propuestas no van más lejos que las tesis de Katz sobre la dependencia de las condicionales de un verbo abstracto en la estructura profunda, que tampoco es nada nuevo, sino una simple formulación lógica (que podría haber sido postulada, por Aristóteles) y que exige la introducción de si por transformación. Quizá lo importante esté en que podamos aceptar, como dice Rivero (art. cit., p. 100), "que el si condicional no es un complementador introducido a nivel de estructura subyacente en las oraciones en que aparece", lo cual engarza directamente con el tema de la subordinación.

La segunda parte del trabajo de M. L. Rivero, en donde se defiende la coordinación entre los dos elementos de la oración condicional, nos parece más aceptable, como, en general, el que se consideren, al menos en buena parte, las subordinadas adverbiales tradicionales como coordinadas. Quizás no sería demasiado difícil llegar ahí a 
un acuerdo entre descriptivistas, estructuralistas y generativistas, aunque no falten voces modernas que discrepen, como la de Thümmel ${ }^{22}$, quien cree que no pueden ser coordinadas, a partir de los ejemplos alemanes, donde sonst ('sino') y andernfalls ('en el caso contrario'), que los gramáticos alemanes consideran coordinadas, no son intercambiables con las típicas condicionales wenn, falls, sofern, wofen, subordinadas, por tanto.

En resumen, creemos que el amplio estudio pancrónico para el que las condicionales se prestan especialmente, podría conceder atención a cuatro puntos básicos: en el terreno histórico a la evolución del esquema temporal-modal del latín al castellano, y al establecimiento del sistema medieval, aparentemente roto en el período clásico por un crecimiento incontrolado de la forma -RA; en el terreno de la descripción, a la tendencia a la simetría de las dos partes del período, lo que también favorece la interpretación coordinada, y, por último, en el plano de la explicación, a los problemas subyacentes.

Francisco Marcos Marín

Universidad de Valladolid.

\section{LA GONSTRUCGIONN DEL CIFAR}

Dos largas secciones del Cifar - libros III y IV- parecen presentarse como irreductibles a todo criterio de unidad ${ }^{1}$. El libro III (Castigos del Rey Menton) es un tratado didáctico-moral que irrumpe en medio de la historia: la calidad de su materia, doctrinal y abstracta, no parece encajar sin dificultad en la narración. El libro IV (Hechos de Roboán) nos devuelve al mundo de la historia y de la acción, pero ésta aparece ahora organizada alrededor de un nuevo personaje. Quienes ven en el tratado doctrinal de los Castigos una quiebra en la estruc-

22 Wolfan ThüмmeL, Vorüberlegungen zu einer Textgrammatik. Koordination und Subordination in der generativen Transformationsgrammatik, Stuttgart, 1970, (mimeografía del original, 191 pp.).

1 Los trabajos de Justina Ruiz Conde (El amor y el matrimonio secreto en las novelas de caballerias, Madrid, 1948), Kenneth Scholberg ("The structure of the Caballero Cifar", $M L N$ 79, 1964, 113-134) y James Burke (History and vision. The figural structure of the "Libro del Cauallero Zifar", Londres, 1972) apuntan ya en otra dirección y señalan una cantidad de elementos que testimonian en el Cifar una fuerte unidad de composición. Burke propone la hipótesis de que en la obra pueden distinguirse dos estratos: uno exterior, primario, aparente, el de la historia, con un significado evidente para cualquier lector; y otro secundario y alegórico. Con respecto a este estrato secundario Burke resume asi su tesis: "I believe that the adventures of Zifar and Roboan fulfil a typological formula which begins in the Old Testament, is modified and given its strongest emphasis in the life of Christ, and which must recur in the lives of certain devout Christians until the end of time (p. 3)". Burke concentra su estudio en este segundo nivel espiritual, pero no aclara la unidad de la obra en el plano de la historia. Sin recurrir a la postulación de esc segundo estrato alegórico, bastante dudoso, el problema de 